\title{
Isolated cortical vein thrombosis: case series
}

\author{
Ranjodh Singh, BPhil, BSc, ${ }^{1}$ William P. Cope, BSc, ${ }^{1}$ Zhiping Zhou, MD, PhD, ${ }^{1}$ \\ Michelle E. De Witt, MD, ${ }^{3}$ John A. Boockvar, MD, ${ }^{3}$ and Apostolos J. Tsiouris, MD²
}

\begin{abstract}
Departments of ${ }^{1}$ Neurological Surgery and ${ }^{2}$ Radiology, Weill Cornell Medical College, NewYork-Presbyterian Hospital; and ${ }^{3}$ Department of Neurosurgery, Lenox Hill Hospital, New York, New York
\end{abstract}

\begin{abstract}
OBJECT Isolated cortical vein thrombosis (ICVT) accounts for less than $1 \%$ of all cerebral infarctions. ICVT may cause irreversible parenchymal damage, rendering early and accurate diagnosis critical. This case series and literature review presents the clinical and radiological findings in 7 patients with ICVT, and highlights risk factors and imaging modalities that may be most beneficial in rendering an accurate and timely diagnosis.

METHODS Patients with CT and MRI findings consistent with ICVT examined between January 2011 and June 2014 were included in this retrospective review.

RESULTS Seven patients ( 5 females, 2 males), ranging in age from 11 months to 34 years, met the inclusion criteria. The most common clinical presentations were headaches $(n=4)$ and seizures $(n=3)$. The most common comorbidities noted in these patients were hypercoagulable states $(n=4)$ and intracranial hypotension $(n=3)$. Five patients had intraparenchymal involvement. CT suggested the correct diagnosis in 4 patients, and MRI confirmed the diagnosis in all 7 patients. All patients who received anticoagulation therapy $(n=5)$ experienced complete resolution of their symptoms.

CONCLUSIONS The majority of these patients were adult females, consistent with published data. Seizures and headaches were the most common presenting symptoms. Hypercoagulable state and intracranial hypotension, both known risk factors for thrombosis, were the most commonly noted ICVT risk factors. Intraparenchymal involvement was prevalent in nearly all ICVT cases and presented as vasogenic edema, early intraparenchymal hemorrhage, or hemorrhagic venous infarction. Susceptibility-weighted imaging was the most sensitive imaging technique in diagnosing ICVT.
\end{abstract}

http://thejns.org/doi/abs/10.3171/2014.9.JNS141813

KEY WORDS intraparenchymal hemorrhage; isolated cortical vein thrombosis; magnetic resonance imaging; susceptibility-weighted imaging; venous infarction; vascular disorders

I SOLATED cortical vein thrombosis (ICVT) is the thrombosis of 1 or more cerebral cortical veins without occlusion of the major dural venous sinuses or the deep cerebral veins. ICVT accounts for less than $1 \%$ of all cerebral infarctions and has only been reported in case reports and small patient series. 1,5,9,24,25,29,35,47 ICVT has a good prognosis if diagnosed and treated early with anticoagu-

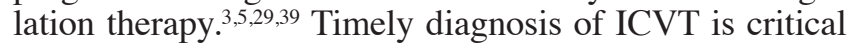
due to the potential for permanent parenchymal damage, which occurs due to vasogenic edema from venous congestion, intraparenchymal hemorrhage (IPH), and venous infarction. ${ }^{22}$ However, ICVT diagnosis remains challenging due to cortical vein anatomical variations, ${ }^{27,42}$ a nonspecific clinical presentation, ${ }^{20,22,29,35,40,47}$ and the lack of a gold-standard imaging modality. ${ }^{5,24,44}$ We present clinical and radiological findings from 7 patients with ICVT to aid clinicians in early and accurate diagnosis of ICVT.

\section{Methods}

This study was approved by the Institutional Review Board at Weill Cornell Medical College. Patients were selected retrospectively from our case database from January 2011 through June 2014 if they had ICVT findings on CT or MRI. MRI included T1-weighted imaging with and without contrast, T2-weighted imaging, T2-weighted FLAIR, susceptibility-weighted imaging (SWI), and diffusionweighted imaging (DWI). Some patients also underwent CT angiography (CTA) and venography (CTV), and MR venography (MRV). All imaging findings were reviewed

ABBREVIATIONS CTA = CT angiography; CTV = CT venography; DWI = diffusion-weighted imaging; $\mathrm{GRE}=$ gradient-recalled echo; ICVT = isolated cortical vein thrombosis; IPH = intraparenchymal hemorrhage; MRV = MR venography; $\mathrm{SAH}$ = subarachnoid hemorrhage; $\mathrm{SDH}$ = subdural hemorrhage; SPGR = spoiled gradient recalled echo; SWI = susceptibility-weighted imaging.

SUBMITTED August 8, 2014. ACCEPTED September 23, 2014.

INCLUDE WHEN CITING Published online March 20, 2015; DOI: 10.3171/2014.9.JNS141813.

DISCLOSURE The authors report no conflict of interest concerning the materials or methods used in this study or the findings specified in this paper. 


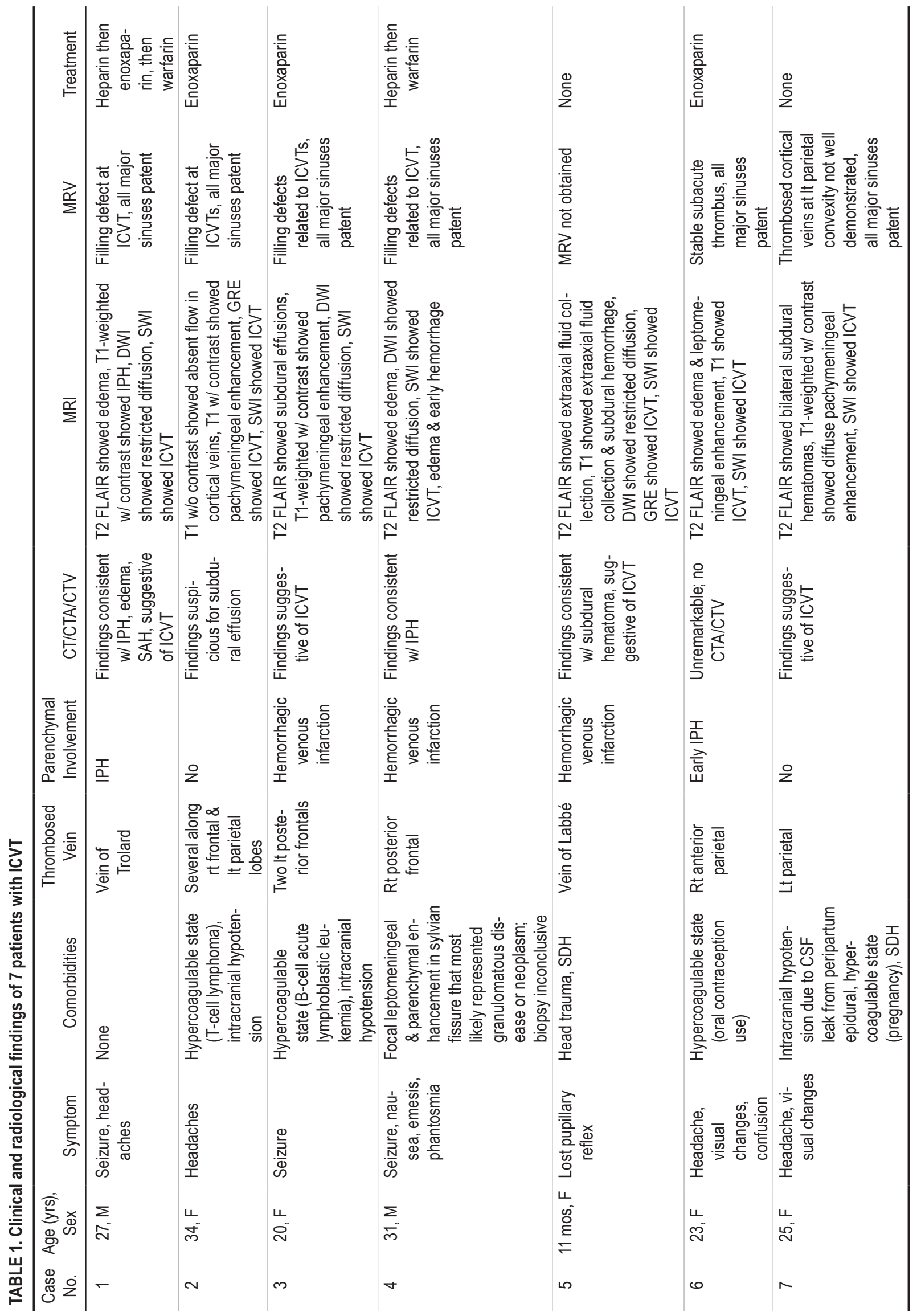




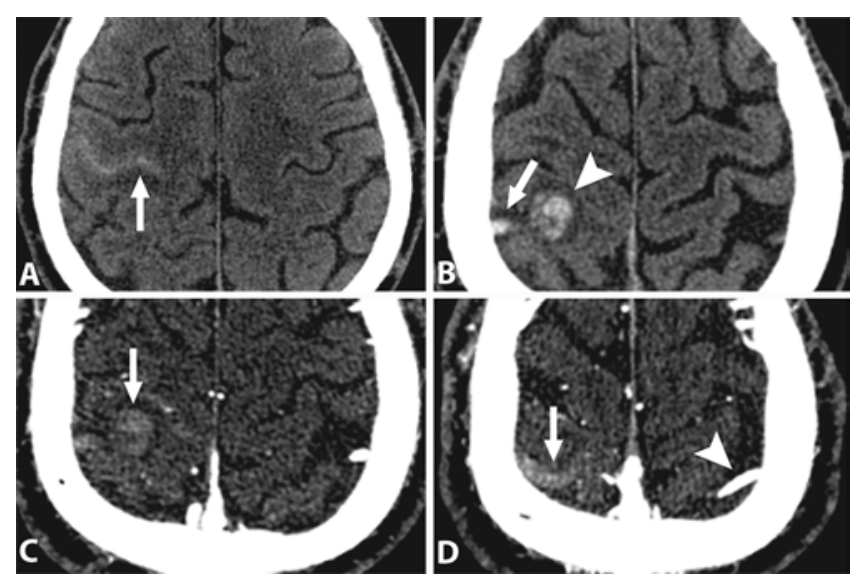

FIG. 1. Axial CT scan without contrast (A) demonstrates abnormal hyperdensity in the right central sulcus (arrow) consistent with SAH. Axial CT scan without contrast (B) also shows a focal hyperdensity in the right postcentral gyrus consistent with IPH (arrowhead), with mild associated edema, and a curvilinear hyperdensity along the lateral postcentral sulcus (arrow) just posterior to the hematoma representing the ICVT. Axial CTA (C) shows a focal hyperdensity in the right postcentral gyrus (arrow) consistent with IPH. Adjacent to the IPH on the CTA (D), a hyperdense curvilinear structure (arrow) is visible that does not enhance like the normal contralateral cortical vein (arrowhead), indicative of ICVT.

by a board-certified diagnostic radiologist with a certificate of added qualification in neuroradiology (A.J.T.).

\section{Results}

\section{Clinical Presentation}

Seven patients met the inclusion criteria (Table 1). One patient was 11 months old (female) and the others ranged from 20 to 34 years old (4 females, 2 males; median age 25 years). Headaches $(n=4)$ and seizures $(n=3)$ were the most common presenting symptoms. The most common comorbidities were hypercoagulable state $(n=4)$ and intracranial hypotension $(n=3)$. Most patients $(n=5)$ re-
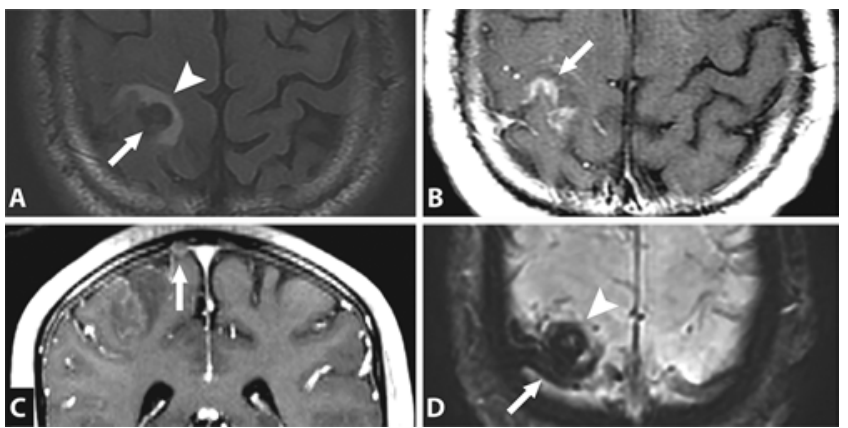

FIG. 2. On axial MR images, IPH is (A) T2 hypointense (arrow) with surrounding vasogenic edema (arrowhead) and (B) hyperintense on T1-weighted imaging (arrow), most consistent with early subacute hemorrhage. Postcontrast coronal T1-weighted SPGR (C) shows an intraluminal filling defect on MRV as the vein of Trolard enters the superior sagittal sinus (arrow), likely indicative of ICVT. Axial SWI (D) demonstrates increased tubular susceptibility (arrow), consistent with ICVT, and increased susceptibility adjacent to the ICVT (arrowhead), representing an IPH.

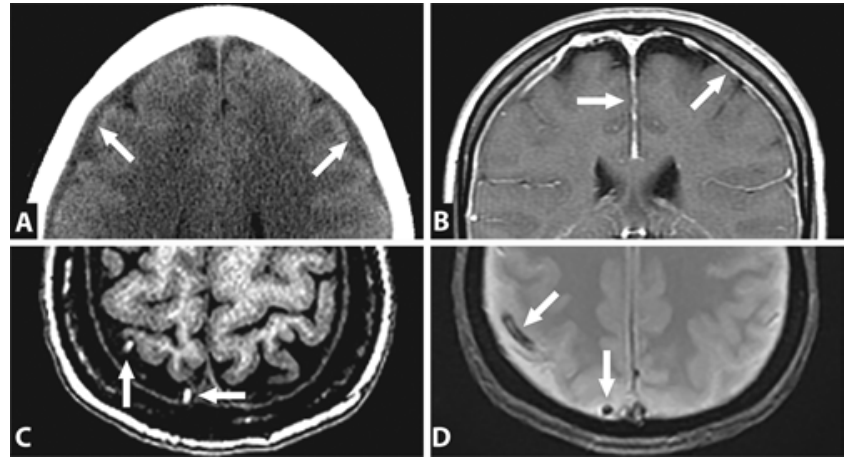

FIG. 3. Axial CT scan (A) shows small bifrontal subdural collections (arrows), and postgadolinium T1-weighted MRI (B) depicts diffuse pachymeningeal enhancement (arrows) related to intracranial hypotension believed to be secondary to a CSF leak from frequent lumbar punctures. Axial T1-weighted SPGR image (C) demonstrates abnormal intraluminal T1 hyperintensity (arrows) that correlates with increased susceptibility on the GRE sequence (D; arrows), most compatible with subacute cortical vein thrombosis.

ceived anticoagulation therapy and experienced resolution of symptoms.

\section{Radiological Findings}

All 7 patients underwent conventional anatomical CT and MRI studies (Figs. 1-7). CT suggested ICVT in 4 patients, which was observed as either a hyperdense cord sign or dot sign on CT scans (Figs. 1B, 4B, and 6A).

MRI confirmed ICVT in all 7 cases. Specifically, SWI accurately identified ICVT in all 7 cases as an increased tubular susceptibility in the thrombosed cortical veins (Figs. 2D, 4C, 5A, and 7B); gradient-recalled echo (GRE) identified ICVT in 2 patients (Figs. 3D and 6E), and T1weighted spoiled gradient recalled echo (SPGR) imaging identified ICVT in 3 cases (Figs. 3C and 7D).

CTA and/or CTV was performed in 2 patients and helped confirm the diagnosis of ICVT, presenting as nonopacification of the affected cortical vein (Figs. 1C, 1D, $6 \mathrm{~B}$, and $6 \mathrm{C})$. MRV was performed in 6 patients and identified ICVT as lack of venous flow-related enhancement and/or filling defects (Figs. 2C and 4E).

Intraparenchymal hemorrhage $(\mathrm{n}=2$; Figs. $1 \mathrm{~B}, 1 \mathrm{C}, 2 \mathrm{~A}$, $2 \mathrm{~B}, 2 \mathrm{D}$, and $7 \mathrm{E})$ and hemorrhagic infarcts $(\mathrm{n}=3$; Figs. 4D, $5 \mathrm{~B}$, and $5 \mathrm{D}$ ) were the most common types of parenchymal involvement. Two patients had associated subdural hemorrhages (SDHs). T2-weighted imaging and T2-weighted FLAIR were useful in identifying parenchymal edema (Figs. 2A and 7F) and venous congestion (Fig. 7G) associated with ICVT in all cases with parenchymal involvement. Intracranial hypotension was visualized as subdural effusions (Fig. 3A and 4A) and diffuse pachymeningeal enhancement (Fig. 3B).

\section{Discussion}

ICVT is commonly misdiagnosed due to developmental anatomical variations of the cortical veins, nonspecific clinical presentation, and lack of a gold-standard imaging modality. In this paper we present a case series to high- 

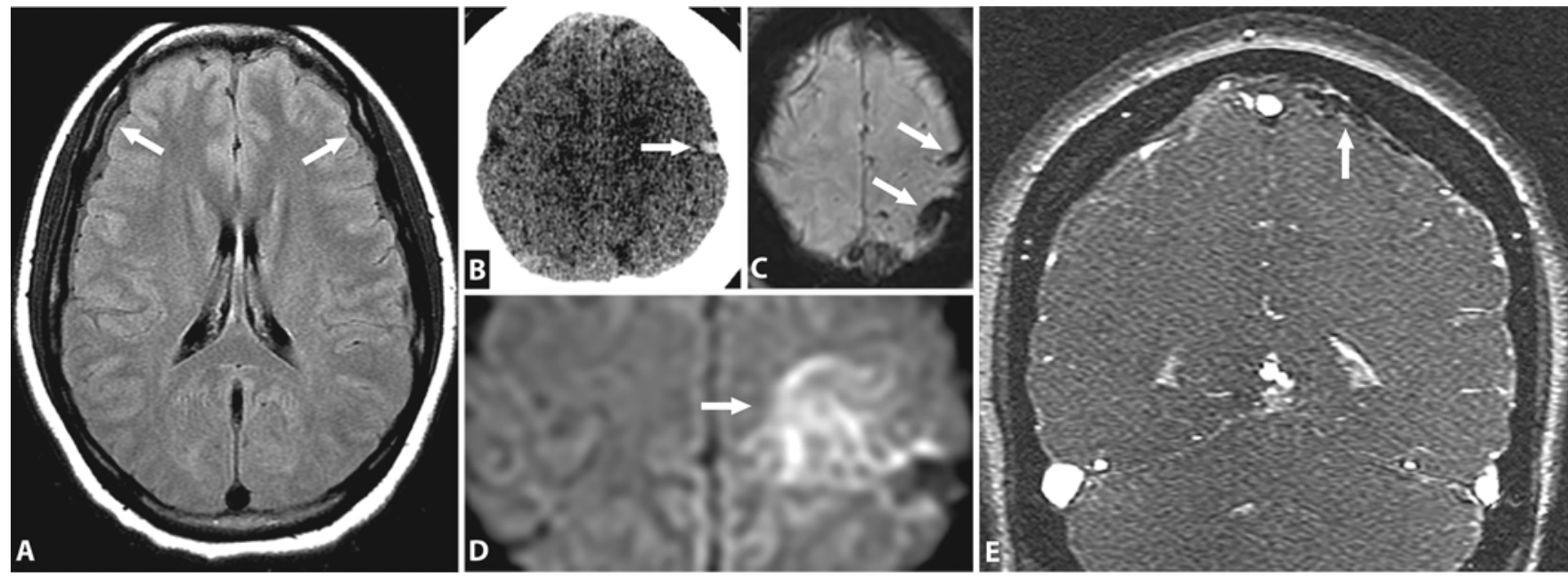

FIG. 4. Axial T2-weighted FLAIR image (A) shows a small bilateral collection (arrows) related to intracranial hypotension, believed to be secondary to a CSF leak from frequent lumbar punctures. An axial CT scan (B) demonstrates a tubular hyperdensity at the left vertex, suspicious for an ICVT. Axial SWI (C) shows increased tubular susceptibility in 2 cortical veins over the left frontoparietal cortex, confirming the diagnosis of ICVT, associated with restricted diffusion (arrow) on DWI (D), indicating early venous infarction, and absence of flow (arrow) on coronal MRV (E).

light the clinical presentation and radiological findings of ICVT. The median age of our patients was 25 years. This is consistent with published data that places young adults (those $<40$ years old) at the highest risk of ICVT. ${ }^{23}$ An 11 -month-old girl had an ICVT in our case series. This is the youngest case reported in the literature. The youngest ICVT cases reported previously occurred in two 14-yearold patients. ${ }^{20,43}$ There was a female predominance in this
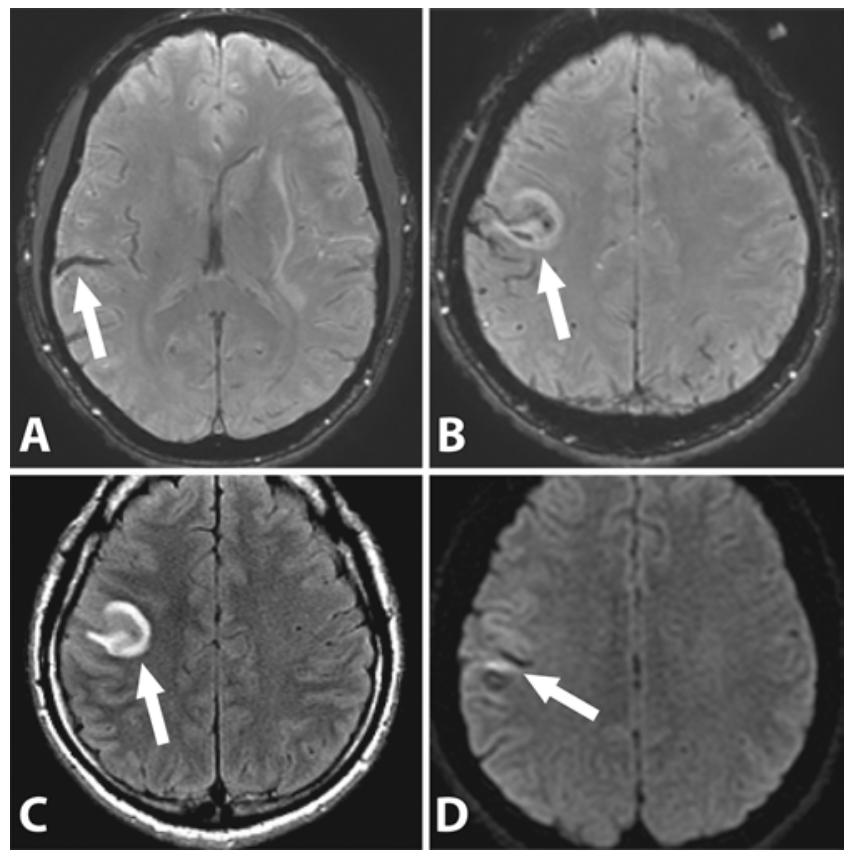

FIG. 5. Axial SWI (A) shows increased tubular susceptibility (arrow) consistent with ICVT, with associated early hemorrhage and edema (B, arrow). The edema is T2 hyperintense (C, arrow), leading to venous congestion and venous infarction with restricted diffusion (arrow) on DWI (D). case series, which is also consistent with previously published data. 22,23

Patients with ICVT may present with a variety of symptoms, including focal or generalized seizures, headaches, hemiparesis, sensory disturbances, and other focal deficits, often without signs of elevated intracranial pressure. ${ }^{9}, 14,15,18,22,29,30,35,47$ Headaches and seizures were the most common clinical symptoms among the 7 patients in this case series, which is consistent with historical data. $^{20,22,29,35,47}$

Common risk factors for ICVT include: 1) a hypercoagulable state due to, for example, oral contraceptive use, postpartum status, or hematological malignancies; 2) head trauma; 3) intracranial hypotension; and 4) infections of the ear, nose, and throat. ${ }^{20,22,29,35,40,47}$ Five patients had a least 1 risk factor for ICVT; of these 5 patient, 3 had two risk factors for ICVT.

Three patients in this case series had intracranial hypotension as a result of CSF leakage from lumbar punctures performed for intrathecal chemotherapy administration or an intrapartum analgesic. Reduction in CSF decreases brain buoyancy and induces a downward shift of the brain, causing pachymeningeal inflammation and mechanical distortion of the cortical veins, which activates the endogenous blood coagulation pathway leading to thrombosis. $1,2,38,48$

Four patients were considered to be in a hypercoagulable state. One patient was using oral contraception, which increases risk for venous thrombosis through decreased function of the endogenous anticoagulation pathway. ${ }^{4,31,41,45}$ One patient was pregnant and 2 others had hematological malignancies, both conditions that are associated with a hypercoagulable state..$^{8,11,12,19}$ In 2 patients no known risk factor was identified, which has been reported in as many as $25 \%$ of patients with ICVT. ${ }^{22,40}$

Five patients with ICVT had intraparenchymal involvement. The primary underlying mechanism is likely an 


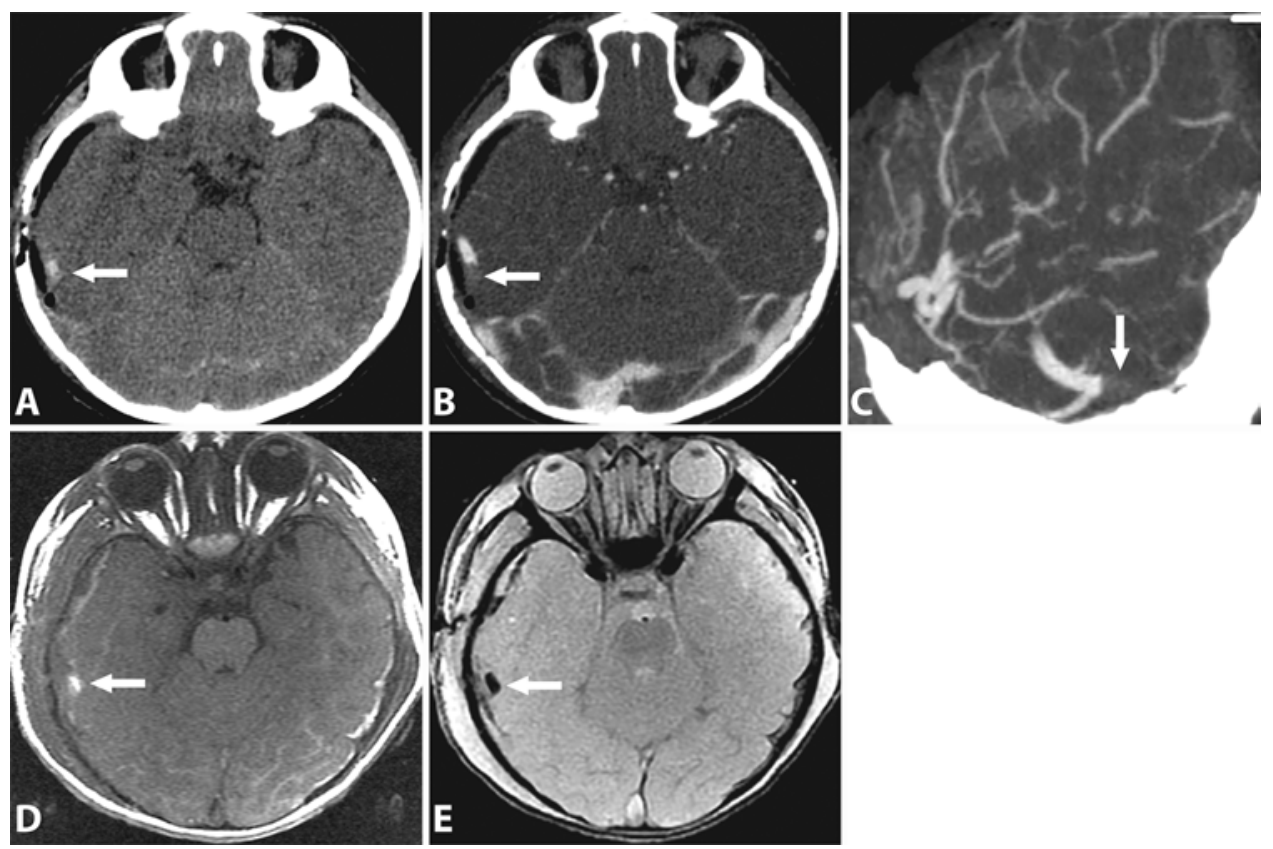

FIG. 6. Axial CT scan without contrast (A) shows a hyperdense tubular structure (arrow), which corresponds to a filling defect on CTA (B, arrow) and sagittal maximal intensity projection reconstructed CTA (C, arrow), suspicious for ICVT. T1-weighted MRI (D) demonstrates abnormal intraluminal T1 hyperintensity (arrow) that correlates with increased susceptibility on the GRE sequence (E, arrow), consistent with ICVT of the vein of Labbé.

increase in venous pressure due to ICVT, which causes edema and venous congestion, resulting in a hemorrhagic infarction. The increase in venous pressure also likely decreases arterial perfusion pressure, causing cell death. Both vasogenic and cytotoxic edema patterns may coexist. $22,32,36,49$

ICVT has been described as having a good prognosis if diagnosed early and anticoagulant therapy is initi- ated. ${ }^{3,5,29,39}$ Our experience supports this, with 5 patients in this case series experiencing resolution of symptoms after receiving anticoagulant therapy. In 2 patients, anticoagulation therapy was not administered due to concern over the potential exacerbation of severe SDHs.

Because clinical presentation is nonspecific, imaging studies play a critical role in ICVT diagnosis. It is important to note that while cerebral angiography was previ-

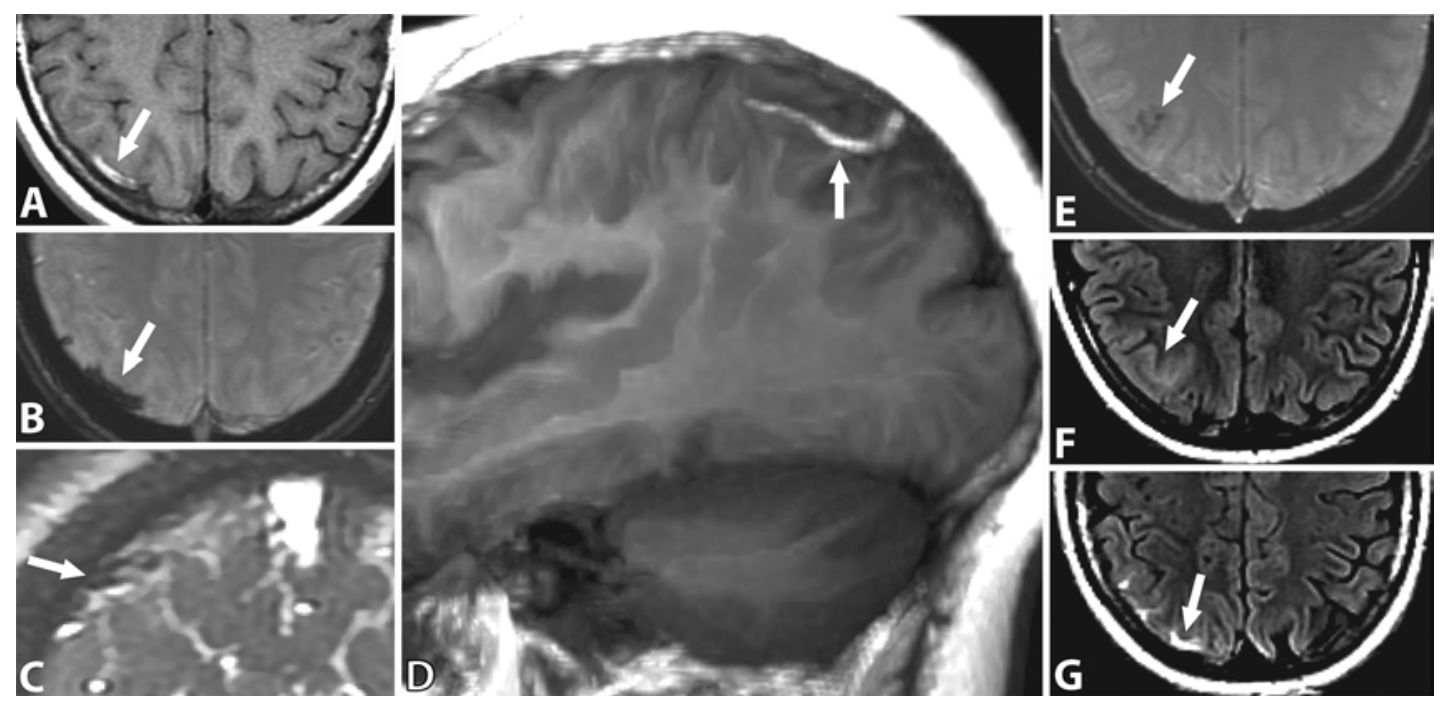

FIG. 7. Axial T1-weighted MRI (A) demonstrates abnormal intraluminal T1 hyperintensity (arrow) that correlates with increased susceptibility on SWI (B, arrow) and with absence of flow-related enhancement (arrow) on GRE time-of-flight coronal MRV (C) consistent with ICVT. Maximal intensity projection reconstruction of the sagittal T1 SPGR sequences (D) better demonstrates the subacute intraluminal ICVT (arrow). SWI (E) shows early IPH (arrow) in the right inferior parietal lobe associated with early parenchymal edema (F, arrow) and venous congestion (G, arrow) on T2-weighted FLAIR imaging. 
ously the gold standard in diagnosing ICVT, it is no longer commonly performed due to availability, sensitivity, and the superior safety profile of CT, CTA/CTV, MRI, and MRV., ${ }^{6,21,35}$ Cerebral angiography is, however, still considered in cases in which the imaging results are inconclusive and when an endovascular procedure is being considered. In our case series, imaging findings on head CT suggested ICVT in 4 patients. Previously published reports show that head CT findings are often nonspecific and may appear normal in more than $30 \%$ of cases. ${ }^{22}$ ICVT often presents on CT as IPH or edema and thus suspicion is warranted when such pathologies are observed without clear etiologies. ${ }^{26,46} \mathrm{CTA} / \mathrm{CTV}$ was performed in 2 patients, but ICVT was already diagnosed in both of these cases from CT scans and therefore the CTA/CTV added no new information regarding the diagnosis.

The American Heart Association and American Stroke Association recommend obtaining MRI and MRV in cases of suspected ICVT. ${ }^{35}$ SWI was the most sensitive in diagnosing ICVT in our case series, which is consistent with previously published studies. $., 13,17,35,44$ Susceptibility contrast arises in MRI from perturbations in the external magnetic field due to local variations in magnetic properties of biological tissue..$^{10}$ Most biological tissues are either diamagnetic or paramagnetic, depending on the atomic structure of the tissue, the number of paired or unpaired electrons, and the alterations in the electron orbits. ${ }^{34,37}$ SWI uses phase information to enhance contrast between tissues with different susceptibilities, ${ }^{16}$ rendering it more sensitive than standard GRE. Blood and its breakdown products produce diamagnetic and paramagnetic effects, ${ }^{28}$ which can be detected with great sensitivity using SWI, making it ideal for accurately identifying ICVT.

While MRV was obtained in 6 patients, ICVT was already diagnosed in all of these cases on MRI, and therefore the MRV added no new information regarding the ICVT diagnosis. A recent review also concluded that MRV results are neither objective nor definitive, and their role in ICVT diagnosis is either supportive or useless. ${ }^{20}$ In this case series, MRV was most useful in confirming the patency of the venous sinuses and deep cerebral veins and determining recanalization of the ICVT at follow-up.

\section{Conclusions}

With the increasing use of newer and improved imaging techniques, the prevalence of ICVT diagnosis has increased, raising suspicion that perhaps ICVT is more

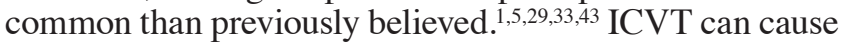
irreversible intraparenchymal damage if not diagnosed early and treated promptly. This case series underscores the importance of obtaining proper imaging, namely SWI, in cases with clinical symptoms and risk factors suggestive of ICVT, and in cases with intraparenchymal hemorrhage, venous congestion, and/or venous infarction of unclear origin.

\section{References}

1. Albayram S, Kara B, Ipek H, Ozbayrak M, Kantarci F: Isolated cortical venous thrombosis associated with intracranial hypotension syndrome. Headache 49:916-919, 2009
2. Albayram S, Tasmali KM, Gunduz A: Can spontaneous intracranial hypotension cause venous sinus thrombosis? J Headache Pain 8:200-201, 2007

3. Bittencourt LK, Palma-Filho F, Domingues RC, Gasparetto EL: Subarachnoid hemorrhage in isolated cortical vein thrombosis: rare presentation of an unusual condition. Arq Neuropsiquiatr 67:1106-1108, 2009

4. Blickstein D, Blickstein I: Oral contraception and thrombophilia. Curr Opin Obstet Gynecol 19:370-376, 2007

5. Boukobza M, Crassard I, Bousser MG, Chabriat H: MR imaging features of isolated cortical vein thrombosis: diagnosis and follow-up. AJNR Am J Neuroradiol 30:344-348, 2009

6. Bousser MG: Cerebral venous thrombosis: diagnosis and management. J Neurol 247:252-258, 2000

7. Casey SO, Alberico RA, Patel M, Jimenez JM, Ozsvath RR, Maguire WM, et al: Cerebral CT venography. Radiology 198:163-170, 1996

8. Chan WS, Rey E, Kent NE, Chan WS, Kent NE, Rey E, et al: Venous thromboembolism and antithrombotic therapy in pregnancy. J Obstet Gynaecol Can 36:527-553, 2014

9. Chang R, Friedman DP: Isolated cortical venous thrombosis presenting as subarachnoid hemorrhage: a report of three cases. AJNR Am J Neuroradiol 25:1676-1679, 2004

10. Duyn J: MR susceptibility imaging. J Magn Reson 229:198207, 2013

11. Falanga A, Marchetti M: Thrombosis in myeloproliferative neoplasms. Semin Thromb Hemost 40:348-358, 2014

12. Falanga A, Russo L, Milesi V: The coagulopathy of cancer. Curr Opin Hematol 21:423-429, 2014

13. Fellner FA, Fellner C, Aichner FT, Mölzer G: Importance of T2*-weighted gradient-echo MRI for diagnosis of cortical vein thrombosis. Eur J Radiol 56:235-239, 2005

14. Ferro JM, Bacelar-Nicolau H, Rodrigues T, Bacelar-Nicolau L, Canhão P, Crassard I, et al: Risk score to predict the outcome of patients with cerebral vein and dural sinus thrombosis. Cerebrovasc Dis 28:39-44, 2009

15. Filippidis A, Kapsalaki E, Patramani G, Fountas KN: Cerebral venous sinus thrombosis: review of the demographics, pathophysiology, current diagnosis, and treatment. Neurosurg Focus 27(5):E3, 2009

16. Haacke EM, Xu Y, Cheng YC, Reichenbach JR: Susceptibility weighted imaging (SWI). Magn Reson Med 52:612-618, 2004

17. Idbaih A, Boukobza M, Crassard I, Porcher R, Bousser MG, Chabriat H: MRI of clot in cerebral venous thrombosis: high diagnostic value of susceptibility-weighted images. Stroke 37:991-995, 2006

18. Jacobs K, Moulin T, Bogousslavsky J, Woimant F, Dehaene I, Tatu L, et al: The stroke syndrome of cortical vein thrombosis. Neurology 47:376-382, 1996

19. James AH: Venous thromboembolism in pregnancy. Arterioscler Thromb Vasc Biol 29:326-331, 2009

20. Kitamura Y, Hara K, Tsunematsu K: Isolated superficial sylvian vein thrombosis with long cord sign: case report and review of the literature. Neurol Med Chir (Tokyo) 54:253259,2014

21. Lafitte F, Boukobza M, Guichard JP, Hoeffel C, Reizine D, Ille O, et al: MRI and MRA for diagnosis and follow-up of cerebral venous thrombosis (CVT). Clin Radiol 52:672-679, 1997

22. Leach JL, Fortuna RB, Jones BV, Gaskill-Shipley MF: Imaging of cerebral venous thrombosis: current techniques, spectrum of findings, and diagnostic pitfalls. Radiographics 26 Suppl 1:S19-S43, 2006

23. Linn J, Brückmann H: Cerebral venous and dural sinus thrombosis: state-of-the-art imaging. Clin Neuroradiol 20:25-37, 2010

24. Linn J, Michl S, Katja B, Pfefferkorn T, Wiesmann M, Hartz $S$, et al: Cortical vein thrombosis: the diagnostic value of 
different imaging modalities. Neuroradiology 52:899-911, 2010

25. Masuhr F, Mehraein S: Cerebral venous and sinus thrombosis: patients with a fatal outcome during intravenous doseadjusted heparin treatment. Neurocrit Care 1:355-361, 2004

26. Morris JG, Fisher M, Carandang RA: Cortical vein thrombosis as a mimic for isolated cortical subarachnoid hemorrhage and transient ischemic attack. Case Rep Neurol 2:63-68, 2010

27. Oka K, Rhoton AL Jr, Barry M, Rodriguez R: Microsurgical anatomy of the superficial veins of the cerebrum. Neurosurgery 17:711-748, 1985

28. Parizel PM, Makkat S, Van Miert E, Van Goethem JW, van den Hauwe L, De Schepper AM: Intracranial hemorrhage: principles of CT and MRI interpretation. Eur Radiol 11:1770-1783, 2001

29. Rathakrishnan R, Sharma VK, Luen TH, Chan BP: The clinico-radiological spectrum of isolated cortical vein thrombosis. J Clin Neurosci 18:1408-1411, 2011

30. Renowden S: Cerebral venous sinus thrombosis. Eur Radiol 14:215-226, 2004

31. Rosing J, Curvers J, Tans G: Oral contraceptives, thrombosis and haemostasis. Eur J Obstet Gynecol Reprod Biol 95:193-197, 2001

32. Röther J, Waggie K, van Bruggen N, de Crespigny AJ, Moseley ME: Experimental cerebral venous thrombosis: evaluation using magnetic resonance imaging. J Cereb Blood Flow Metab 16:1353-1361, 1996

33. Sagduyu A, Sirin H, Mulayim S, Bademkiran F, Yunten N, Kitis O, et al: Cerebral cortical and deep venous thrombosis without sinus thrombosis: clinical MRI correlates. Acta Neurol Scand 114:254-260, 2006

34. Saini S, Frankel RB, Stark DD, Ferrucci JT Jr: Magnetism: a primer and review. AJR Am J Roentgenol 150:735-743, 1988

35. Saposnik G, Barinagarrementeria F, Brown RD Jr, Bushnell $\mathrm{CD}$, Cucchiara B, Cushman M, et al: Diagnosis and management of cerebral venous thrombosis: a statement for healthcare professionals from the American Heart Association/ American Stroke Association. Stroke 42:1158-1192, 201

36. Schaller B, Graf R, Sanada Y, Tolnay M, Rosner G, Wienhard $\mathrm{K}$, et al: Hemodynamic changes after occlusion of the posterior superior sagittal sinus: an experimental PET study in cats. AJNR Am J Neuroradiol 24:1876-1880, 2003

37. Schenck JF: The role of magnetic susceptibility in magnetic resonance imaging: MRI magnetic compatibility of the first and second kinds. Med Phys 23:815-850, 1996

38. Schievink WI: Spontaneous spinal cerebrospinal fluid leaks and intracranial hypotension. JAMA 295:2286-2296, 2006

39. Sharma VK, Teoh HL: Isolated cortical vein thrombosis - the cord sign. J Radiol Case Rep 3:21-24, 2009
40. Stam J: Cerebral venous and sinus thrombosis: incidence and causes. Adv Neurol 92:225-232, 2003

41. Stegeman BH, de Bastos M, Rosendaal FR, van Hylckama Vlieg A, Helmerhorst FM, Stijnen T, et al: Different combined oral contraceptives and the risk of venous thrombosis: systematic review and network meta-analysis. BMJ 347:f5298, 2013

42. Tanriverdi T, Al-Jehani H, Poulin N, Olivier A: Superficial anastomotic veins: neurosurgical view depending on 251 craniotomies. Can J Neurol Sci 36:65-71, 2009

43. Thamburaj K, Choudhary A: Hyperintense vessel sign: isolated cortical venous thrombosis after L-asparaginase therapy. Pediatr Radiol 39:757, 2009

44. Urban PP, Müller-Forell W: Clinical and neuroradiological spectrum of isolated cortical vein thrombosis. J Neurol 252:1476-1481, 2005

45. van Hylckama Vlieg A, Helmerhorst FM, Vandenbroucke JP, Doggen CJ, Rosendaal FR: The venous thrombotic risk of oral contraceptives, effects of oestrogen dose and progestogen type: results of the MEGA case-control study. BMJ 339:b2921, 2009

46. Wang YF, Fuh JL, Lirng JF, Chang FC, Wang SJ: Spontaneous intracranial hypotension with isolated cortical vein thrombosis and subarachnoid haemorrhage. Cephalalgia 27:1413-1417, 2007

47. Xue SF, Ma QF, Ma X, Jia JP: Isolated cortical vein thrombosis: a widely variable clinicoradiological spectrum. Eur Neurol 69:331-335, 2013

48. Yildiz OK, Balaban H, Cil G, Oztoprak I, Bolayir E, Topaktas S: Isolated cortical vein thrombosis after epidural anesthesia: report of three cases. Int J Neurosci 120:447-450, 2010

49. Yuh WT, Simonson TM, Wang AM, Koci TM, Tali ET, Fisher DJ, et al: Venous sinus occlusive disease: MR findings. AJNR Am J Neuroradiol 15:309-316, 1994

\section{Author Contributions}

Conception and design: Tsiouris, Singh, Boockvar. Acquisition of data: Tsiouris, Singh, Cope. Analysis and interpretation of data: all authors. Drafting the article: all authors. Approved the final version of the manuscript on behalf of all authors: Tsiouris. Study supervision: Tsiouris, Boockvar. Figure compilation: Zhou.

\section{Correspondence}

Apostolos J. Tsiouris, Department of Radiology, Neuroradiology Section, Weill Cornell Medical College, NewYork-Presbyterian Hospital, 525 E. 68th St., Starr 630C, New York, NY 10021. email: apt9001@med.cornell.edu. 